\section{LINEAR END-SYSTOLIC PRESSURE-VOLUME RELATIONSHIP DURING PULSATILE LEFT VENTRICULAR BYPASS REPRESENTS NATIVE HEART FUNCTION}

This study assessed whether the end-systolic pressure-volume relationship obtained without any interventions during pulsatile left ventricular bypass adequately represents native heart function. In 11 anesthetized Holstein calves, left ventricular pressure was measured with a micromanometer while left ventricular volume was simultaneously calculated from orthogonal left ventricular diameters measured with ultrasonic dimension transducers. End-systolic pressure and volume data were subjected to linear regression analysis to achieve an end-systolic pressure-volume relationship. Data from both caval occlusions and aortic occlusion were used for the control end-systolic pressure-volume relationship (median $r=0.941$, slope $=7.4 \pm$ $0.8 \mathrm{~mm} \mathrm{Hg}$ per milliliter per $100 \mathrm{gm}$ left ventricular weight; mean \pm standard error of the mean). During left atrial-aortic bypass with a Pierce-Donachy pneumatic assist pump in the asynchronous mode, the end-systolic pressure-volume relationships were obtained without interventions to change ventricular loading conditions. During maximal ventricular unloading during full to empty pumping, termed $100 \%$, the resulting narrow range of pressure and volume data did not yield highly linear end-systolic pressure-volume relationships (median $r=0.669$, slope $=4.9 \pm 0.9 \mathrm{~mm}$ Hg per milliliter per $100 \mathrm{gm}$ left ventricular weight). However, at reduced rates off pumping, the end-systolic pressure-volume relationships were considerably linear (80\%, median $r=0.819 ; 60 \%$, median $r=0.868 ; 40 \%$, median $r=0.899)$. Slopes did not significantly differ from control values $(80 \%, 6.9 \pm 1.1 ; 60 \%, 8.2 \pm 1.1 ; 40 \%, 7.8$ \pm 1.1). The end-systolic pressure-volume relationship obtained without exogenous load changes during asynchronous, pulsatile left ventricular bypass represents native left ventricular systolic function. (J THORAC CARDIOvASC SuRG 1995;109:780-6)

Osamu Kawaguchi, MD, John S. Sapirstein, MD, William B. Daily, MD, ${ }^{*}$

Walter E. Pae, MD, FACS, and William S. Pierce, MD, FACS, Hershey, Pa.
Lets

eft ventricular (LV) bypass has become an acLcepted therapy for patients who cannot be weaned from cardiopulmonary bypass. Approximately $45 \%$ of the patients with postcardiotomy cardiogenic shock who are treated with mechanical LV assistance are weaned from the devices, and nearly $50 \%$ of these ultimately survive. ${ }^{1-3}$

An important consideration for appropriate timing of weaning from $\mathrm{LV}$ bypass in patients with postcardiotomy cardiogenic shock involves determi-

From the Division of Cardiothoracic Surgery, Department of Surgery, College of Medicine, and The Milton S. Hershey Medical Center, The Pennsylvania State University, Hershey, Pa.

Received for publication March 10, 1994.

Accepted for publication July 28, 1994.

Address for reprints: William S. Pierce, MD, P.O. Box 850, Department of Surgery, The Milton S. Hershey Medical Center, The Pennsylvania State University, Hershey, PA 17033.

*Department of Surgery, Jewish Hospital, 216 South Kingshighway, St. Louis, MO 63110.

Copyright (C) 1995 by Mosby-Year Book, Inc.

$0022-5223 / 95 \$ 3.00+0 \quad \mathbf{1 2 / 1 / 5 9 4 9 3}$ nation of recovery of native heart function sufficient for maintaining the systemic circulation. Mean aortic pressure, pulmonary arterial pressure, left atrial pressure, ejection fraction, and cardiac index, with and without LV bypass, have been used to assess native heart function. ${ }^{4-7}$ However, because LV bypass affects ventricular loading conditions, most of the conventional, load-dependent hemodynamic parameters are not necessarily appropriate in assessing native heart recovery. Therefore, it has been difficult to accurately determine suitable myocardial recovery without weaning trials.

The linear end-systolic pressure-volume relationship (ESPVR), described by its slope $\left(E_{e s}\right)$ and volume intercept $\left(V_{0}\right)$, has been found to be a load-independent index of ventricular contractile state. ${ }^{8,9}$ For the ESPVR to be extrapolated, interventions that change ventricular loading conditions (preload or afterload) are generally necessary. ${ }^{10-12}$ During asynchronous pulsatile LV bypass, though, LV loading conditions change with variation of the pumping rate and with each pumping cycle. There- 
fore the ESPVR can be generated during asynchronous LV bypass without any other invasive manipulations to alter ventricular loading conditions. The purpose of this study was to evaluate whether the ESPVR generated during asynchronous LV bypass is a good index of native LV contractility.

\section{Materials and methods}

Experimental preparation. A total of 11 Holstein calves weighing from 86 to $112 \mathrm{~kg}$ (average $95 \pm 8 \mathrm{~kg}$ ) was studied. All animals involved in this study received humane care in compliance with the "Principles of Laboratory Animal Care" formulated by the National Society for Medical Research and the "Guide for the Care and Use of Laboratory Animals" prepared by the Institute of Laboratory Animal Research and published by the National Institutes of Health (NIH Publication No. 86-23, revised 1985).

With the animal under halothane anesthesia, endotracheal intubation was performed and ventilation maintained by a volume respirator with $100 \%$ oxygen. After median sternotomy, the left internal thoracic artery was cannulated to monitor aortic pressure. The azygos and hemiazygos veins were ligated. Reversible occluders were placed around the superior and inferior venae cavae.

As illustrated in Fig. 1, pulse-transit ultrasonic dimension crystals ( $4 \mathrm{~mm}$ diameter), which were made of $5 \mathrm{MHz}$ piezoelectric crystals (LTZ-2, Transducer Products, Goshen, Conn.) and coated with epoxy in our laboratory, were placed in the endocardium across the anteroposterior minor axis, septal-free wall minor axis, and base-apex major axis of the LV. The septal transducer was placed into the ventricular septum just to the right of the left anterior descending coronary artery and positioned as near as possible to the LV endocardial surface midway between the anterior and posterior transducers. The base transducer was positioned in the groove between the left sinus of Valsalva and the left atrium overlying the fibrous continuity between the aortic and mitral valves.

A micromanometer-tipped catheter (model SPC-350, Millar Instruments, Inc., Houston, Tex.) was inserted into the LV through the LV free wall to measure LV pressure. A fluid-filled polyethylene catheter was inserted into the $\mathrm{LV}$ in the same manner. The electronic micromanometer in the $\mathrm{LV}$ was connected to a pressure transducer control unit (model TCB-500, Millar Instruments, Inc.) and calibrated and balanced at $37^{\circ} \mathrm{C}$ with a mercury manometer before each experiment. Catheter signal drift was rechecked in vivo by comparison with the LV pressure signal obtained simultaneously from the fluid-filled catheter.

Anticoagulation was achieved with an intravenous heparin injection (300 units $/ \mathrm{kg}$ ). The assist pump outflow cannula was sutured to the ascending aorta, and the $51 \mathrm{~F}$ cannula for assist pump inflow was inserted through a pursestring suture in the left atrial appendage. These cannulas were connected to the inlet and outlet ports of a Pierce-Donachy pneumatic assist pump (100 ml stroke volume) built in our laboratory and LV bypass was initiated.

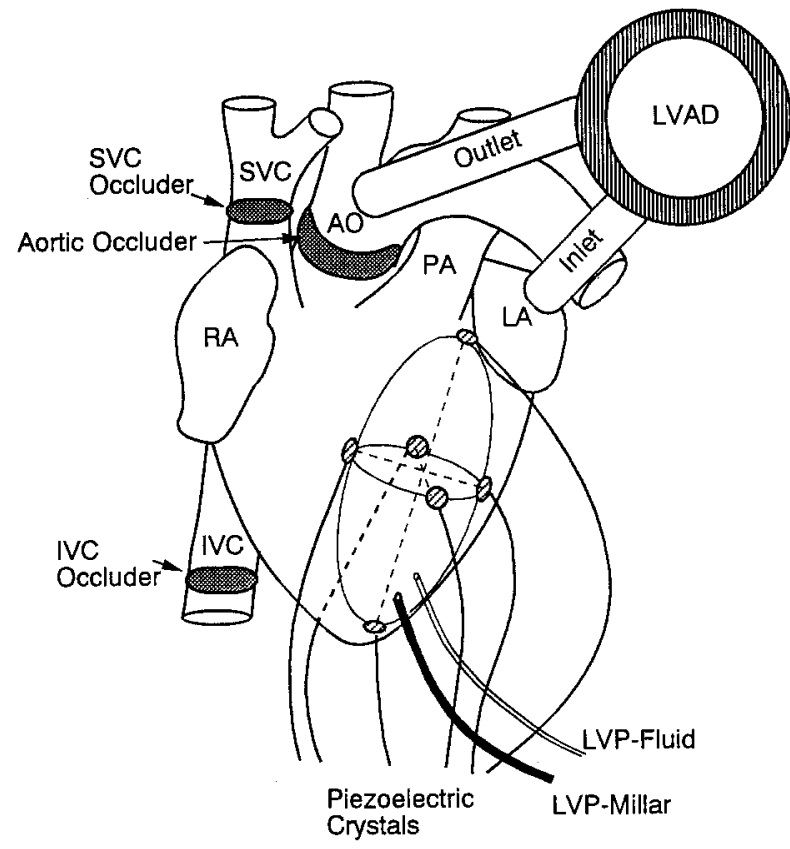

Fig. 1. Schematic diagram of the heart preparation. Three pairs of piezoelectric crystals are implanted endocardially. $A O$, Aorta; Inlet, inlet cannula of $\mathrm{LV}$ assist device from left atrium; $I V C$, inferior vena cava; $L A$, left atrium; $L V A D$, left ventricular assist device; LVP-Millar, catheter-tipped electronic LV pressure monitor; LVPFluid, fluid-filled LV pressure monitor; Outlet, outlet cannula of $\mathrm{LV}$ assist device to ascending aorta; $P A$, pulmonary artery; $R A$, right atrium; $S V C$, superior vena cava.

Digoxin $(0.25 \mathrm{mg}$, intravenously) and lidocaine (2.5 $\mathrm{mg} / \mathrm{min}$, intravenously) were administered to prevent arrhythmia during the preparation. If needed, a constant infusion of epinephrine $(0.4$ to $2.5 \mu \mathrm{g} / \mathrm{kg}$ per minute, intravenously), phenylephrine $(0.3$ to $1.0 \mu \mathrm{g} / \mathrm{kg}$ per minute, intravenously), isoproterenol $(0.002$ to $0.01 \mu \mathrm{g} / \mathrm{kg}$ per minute, intravenously), or a combination thereof, was used in some of the preparations to prevent systemic hypotension or pulmonary hypertension. In all cases, these catecholamines were successfully withdrawn before data collection began. To maintain appropriate ventricular filling throughout the experimental protocol, we made minor adjustments of intravascular blood volume by infusion of whole blood.

Experimental protocol. After the preparation had reached a steady state, baseline pressure and volume measurements were taken by changing preload or afterload during transient occlusions of the venae cavae and then the ascending aorta (5 to 8 seconds for each); these data yielded a control ESPVR (control run) from a wide range of pressure and volume data. Asynchronous pulsatile LV bypass was then brought to a maximal rate, termed $100 \%$, as determined by the pump filling in the full to empty mode, and data were obtained without applying aortic or caval occlusions (LV bypass run). Further LV 
Table I. Hemodynamic changes before and during left atrial-aortic bypass

\begin{tabular}{|c|c|c|c|c|c|}
\hline & \multicolumn{4}{|c|}{ LV bypass: Pump rate (beats $/ \mathrm{min}$ ) $/ \%$ rate } & \multirow{2}{*}{$\frac{\text { Control }}{N A}$} \\
\hline & $71 \pm 9 / 100 \%$ & $57 \pm 7 / 80 \%$ & $43 \pm 6 / 60 \%$ & $28 \pm 4 / 40 \%$ & \\
\hline HR (beats/min) & $100 \pm 4$ & $102 \pm 4$ & $101 \pm 4$ & $100 \pm 4$ & $99 \pm 4$ \\
\hline $\mathrm{EDV}(\mathrm{ml})$ & $90.3 \pm 10.1^{*}$ & $101.5 \pm 10.3^{*}$ & $104.4 \pm 10.7^{*}$ & $109.2 \pm 11.7$ & $118.9 \pm 12.1$ \\
\hline $\mathrm{EDP}(\mathrm{mm} \mathrm{Hg})$ & $6.9 \pm 1.1^{*}$ & $8.9 \pm 1.1^{*}$ & $10.1 \pm 1.1^{*}$ & $11.6 \pm 1.2$ & $14.0 \pm 1.4$ \\
\hline $\mathrm{ESV}(\mathrm{ml})$ & $75.9 \pm 8.5^{*}$ & $85.1 \pm 8.6$ & $86.2 \pm 8.6$ & $87 \pm 8.8$ & $92.2 \pm 9.8$ \\
\hline $\mathrm{ESP}(\mathrm{mm} \mathrm{Hg})$ & $46 \pm 4^{*}$ & $60 \pm 4$ & $64 \pm 4$ & $64 \pm 4$ & $68 \pm 4$ \\
\hline CVP (mm Hg) & $6.7 \pm 1.0$ & $6.4 \pm 0.8$ & $6.4 \pm 0.8$ & $6.6 \pm 0.7$ & $6.8 \pm 0.6$ \\
\hline $\mathrm{mAoP}(\mathrm{mm} \mathrm{Hg})$ & $58 \pm 4$ & $61 \pm 4$ & $59 \pm 3$ & $58 \pm 3$ & $57 \pm 4$ \\
\hline $\mathrm{CO}(\mathrm{L} / \mathrm{min})$ & $6.7 \pm 0.7$ & $7.0 \pm 0.6$ & $7.4 \pm 1.0$ & $7.0 \pm 0.7$ & $6.8 \pm 0.5$ \\
\hline$+\mathrm{dP} / \mathrm{dt}(\mathrm{mm} \mathrm{Hg} / \mathrm{sec})$ & $339 \pm 31^{*}$ & $464 \pm 30^{*}$ & $472 \pm 34^{*}$ & $514 \pm 41$ & $577 \pm 41$ \\
\hline
\end{tabular}

$H R$, Heart rate; $E D V$, end-diastolic volume; $E D P$, end-diastolic pressure; $E S V$, end-systolic volume; $E S P$, end-systolic pressure; $C V P$, central venous pressure; $m A o P$, mean aortic pressure; $C O$, cardiac output; $+d P / d t$, maximal rate of ventricular pressure rise. Mean \pm standard error of the mean is indicated. ${ }^{*} p<0.05$ compared with control value.

bypass data collections were conducted with the pumping: $80 \%, 60 \%$, and $40 \%$ of the maximal pumping rate. Steady-state equilibrium of the preparation was assured before acquisition of each data set by inspection of real-time pressure-volume loops and $\mathrm{E}_{\mathrm{es}}$. The measurements were repeated at an interval of 1 to 2 minutes under the same steady-state conditions to confirm reproducibility of the data.

At the conclusion of the experimental protocol, the animal was put to death. The heart was excised to verify proper positioning of the transducers, and the LV was weighed.

Data analysis. Ultrasonic signals were continuously monitored with a four-channel ultrasonic dimension system (system 6 chassis, Triton Technology, San Diego, Calif.) at a sampling rate of $1302 \mathrm{~Hz}$ and a practical frequency response of 0 to $64 \mathrm{~Hz}$. Minimal resolution was $1 \mathrm{~mm}$. The instantaneous distance between each pair of crystals was calculated according to the velocity of sound in blood $\left(1.58 \times 10^{5} \mathrm{~cm} / \mathrm{sec}\right)$. These dimension signals and the LV pressure signals were sampled at $7 \mathrm{msec}$ intervals for 13 seconds and stored on a floppy disk by a custommade, on-line data acquisition system. Data were also recorded on a multichannel thermal-pen recorder (Gould 3000 series thermal chart recorder, Gould Inc., Cleveland, Ohio).

Data were analyzed with interactive computer software developed in our laboratory. LV volume was calculated from a modified ellipsoidal shell model:

$$
\mathrm{LV} \text { volume }=\pi / 6 \cdot \mathrm{D}_{\mathrm{AP}} \cdot \mathrm{D}_{\mathrm{SL}} \cdot \mathrm{D}_{\mathrm{L}}
$$

where $D_{L}$ is the endocardial base-apex major axis, $D_{A P}$ is the anteroposterior minor axis, and $\mathrm{D}_{\mathrm{SI}}$ is the septal-free wall minor axis orthogonal diameter of the LV. ${ }^{13-16}$ The contractile state of the LV was assessed by ventricular end-systolic elastance, $\mathrm{E}_{\mathrm{es}}{ }^{8,9}$ The ESPVR was generated with linear regression analysis through simultaneous endsystolic pressure and volume data. ${ }^{10-14} \mathrm{E}_{\mathrm{es}}$ was defined as the slope of the ESPVR, and LV unloaded volume $\left(V_{0}\right)$ was determined as the volume intercept of the ESPVR. We determined provisional end-systole at the timing of minimal LV compliance and then used repetitive linear regressions in which the linear estimation of $V_{0}$ was used to determine end-systole as the maximal pressure/( $\mathrm{LV}$ volume $-V_{0}$ ) ratio in each cardiac cycle for a subsequent linear regression. ${ }^{17} \mathrm{We}$ defined end-diastole from electrocardiography. Iteration was continued until $\mathrm{E}_{\mathrm{es}}$ and $\mathrm{V}_{0}$ converged to their respective values. All $\mathrm{E}_{\mathrm{es}}$ values were normalized for $100 \mathrm{gm} \mathrm{LV}$ weight. We also measured maximal rate of pressure rise $(\max \mathrm{dP} / \mathrm{dt}$ ) as one of the traditional measures of LV contractility.

Statistics. Comparisons of variables among the groups were tested by two-way analysis of variance. When analysis of variance showed statistical significance by $F$ test, mean values were compared by the least significant difference method. Probability values smaller than 0.05 were considered statistically significant. Data are presented as mean plus or minus standard error of the mean unless otherwise indicated.

\section{Results}

Table I shows hemodynamic parameters with and without $\mathrm{LV}$ bypass. $\mathrm{LV}$ bypass did not affect heart rate or cardiac output at any rate of pumping. End-diastolic volume and pressure significantly decreased with increases in pumping rate. Central venous pressure and mean aortic pressure remained unchanged. Inasmuch as the maximal rate of pressure rise $(\max \mathrm{dP} / \mathrm{dt})$, considered one index of ventricular contractility, is dependent on end-diastolic volume, it decreased as pumping rate increased.

Fig. 2 shows representative pressure and volume tracings and pressure-volume loops from one animal during LV bypass. Pumping rate was fixed at 80 beats/min. LV pressure and volume tracings varied beat by beat as the asynchrony of timing between native heart ejection and ejection from LV bypass altered preload and/or afterload. From these pressure-volume loops, an ESPVR could be generated by linear regression without any other interventions to alter LV loading conditions. 


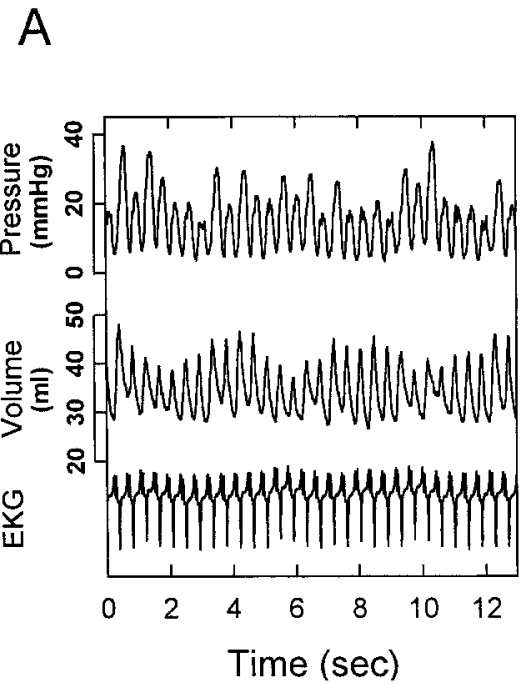

B

Fig. 2. A, Representative time tracings of LV pressure and volume signals and electrocardiogram. B, Pressure-volume loops of the control $(\square)$ and left ventricular bypass $(O)$.

Fig. 3 shows the end-systolic pressure-volume data points and regression line during both control and LV bypass runs for the same heart shown in Fig. 2. As shown in Fig. 3, $A$, a highly linear, control ESPVR was found by altering ventricular loading conditions with vena caval and aortic occlusions. In each varied setting of pumping rate, linearity was evident, and the $E_{e s}$ and $V_{0}$ of the ESPVR were consistent with the $\mathrm{E}_{\text {es }}$ and $\mathrm{V}_{0}$ of the other ESPVRs shown in Fig. 3, $B$ to $E$. This fact is further demonstrated in Fig. 3, $F$, in which all end-systolic pressure-volume data points are superimposed.

Table II averages the results of linear regression analysis for end-systolic pressure-volume data points from all 11 experiments. During maximal pumping $(100 \%)$, highly linear ESPVRs were not generally obtained from the relatively narrow ranges of data. $\mathrm{V}_{0}$ during maximal pumping was significantly lower than $\mathrm{V}_{0}$ values obtained during the other pumping rates or control runs. However, this difference is understandable because of the need to extrapolate $\mathrm{V}_{0}$ from $100 \%$ pumping rate data that had a relatively poor linear fit (median $r=0.669$ ). In contrast, correlation of a linear ESPVR was high during other pumping rates (median $r>0.8$ ); in these runs there were no significant differences from control $\mathrm{E}_{\mathrm{es}}$ and $\mathrm{V}_{0}$.

\section{Discussion}

Complications such as infection, embolus, and renal failure are associated with mechanical LV bypass. Therefore, attempts are made to wean pa- tients from the LV bypass as soon as possible. On the other hand, because use of LV bypass as a bridge to heart transplantation has achieved excellent results, continuation of $\mathrm{LV}$ bypass until subsequent heart transplantation would be another option for the patient with postcardiotomy cardiogenic shock who is dependent on the device and does not have contraindications to transplantation. Therefore, it is important to assess not only whether the patient can be weaned from LV bypass but also whether he can survive without LV bypass. Assessing the propriety of weaning requires an accurate determination of ventricular performance. Because most of the traditional hemodynamic parameters are load dependent, as shown in maximal rate of pressure rise (max $\mathrm{dP} / \mathrm{dt}$ ), hemodynamics have been assessed during transient discontinuance of $\mathrm{LV}$ bypass pumping. Despite such a maneuver, a single hemodynamic parameter does not consistently reflect native heart function, and thus combinations of parameters are needed. ${ }^{5-7}$ Additionally, abrupt cessation of LV assistance can conceivably overload a recovering ventricle and further impair its function.

The slope of the ESPVR, $\mathrm{E}_{\mathrm{es}}$, which we quantified in this study, has been recognized as a load-independent measurement of ventricular performance in both the experimental and clinical settings. ${ }^{14,15} \mathrm{~A}$ wide range of $L V$ pressure and volume data is generally necessary to determine the linear ESPVR, ${ }^{14,15}$ and usually interventions that explicitly change ventricular loading conditions are used to provide this range. Unfortunately, techniques for altering ventricular 

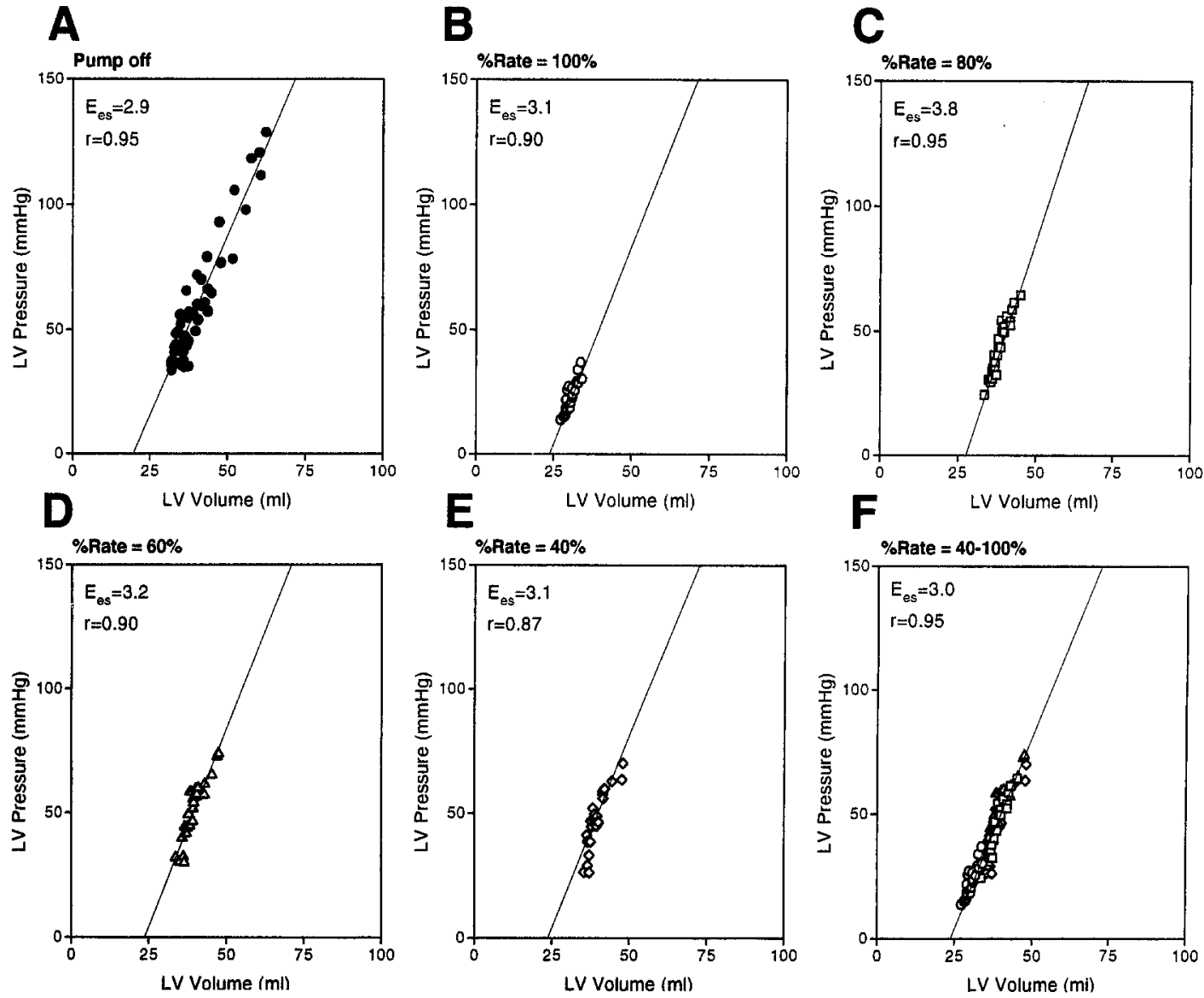

Fig. 3. ESPVRs in the control and during indicated pumping rates of $\mathrm{LV}$ bypass. \% Rate, Percent pumping rate; $E_{\text {es }}$, slope of end-systolic pressure-volume relationship; $r$, correlation coefficient. Linear regression lines are indicated in each panel.

Table II. Results of linear regression of end-systolic pressure and volume

\begin{tabular}{lccccc}
\hline & \multicolumn{4}{c}{ LV bypass: \% Rate } & \multicolumn{2}{c}{ Control } \\
\cline { 2 - 4 } \cline { 2 - 4 } & $100 \%$ & $80 \%$ & $60 \%$ & $40 \%$ & NA \\
\hline Median $r$ & 0.669 & 0.819 & 0.868 & 0.899 & 0.941 \\
$\mathrm{E}_{\mathrm{cs}}(\mathrm{mm} \mathrm{Hg} / \mathrm{ml} / 100 \mathrm{gm} \mathrm{LV})$ & $4.9 \pm 0.9$ & $6.9 \pm 1.1$ & $8.2 \pm 1.1$ & $7.8 \pm 1.1$ & $7.4 \pm 0.8$ \\
$\mathrm{~V}_{0}(\mathrm{ml})$ & $32.7 \pm 8.6^{*}$ & $50.0 \pm 8.4$ & $55.5 \pm 8.4$ & $53.9 \pm 8.7$ & $56.7 \pm 6.4$ \\
\hline
\end{tabular}

$N A$, Not applied; median $r$, median of correlation coefficient; \% rate, percentage of maximal pumping rate. Mean \pm standard error of the mean is indicated. ${ }^{*} p<0.05$ compared with control value.

loading conditions, such as balloon occlusion of the descending aorta or venae cavae, make the measurement of $E_{\text {es }}$ complicated. ${ }^{10-14}$

When LV bypass is operated in an asynchronous, full-to-empty mode, loading conditions of the native ventricle change beat-to-beat because of competition for preload and changing afterload between $\mathrm{LV}$ bypass and the native heart. In theory, therefore, determination of the native heart's ESPVR during asynchronous LV bypass could be made without any interventions to alter ventricular loading conditions. As revealed in Table II, neither a good, linear fit for the ESPVR nor an accurate representation of $E_{\mathrm{es}}$ was generally obtained during $100 \%$ pumping; therefore this slope of the ESPVR may not adequately represent native heart contractility. At lower rates, though, a broader range of pressure-volume data and linearity of the ESPVR were preserved. $E_{\text {es }}$ and $V_{0}$ values were consistent with the control values at the lower rates. As demonstrated by com- 
parison of panels $A$ and $F$ in Fig. 3, pooled pressurevolume data from different pumping rates essentially duplicated $E_{e s}$ and $V_{0}$ obtained in the traditional (control) manner. Thus the LV loadindependent contractility index, $\mathrm{E}_{\mathrm{es}}$, can be faithfully estimated during LV bypass without the risk of myocardial injury precipitated by abruptly stopping the pump.

If the native heart cannot yield systemic pressure and cardiac output sufficient for coronary circulation, then the coronary blood supply essentially depends on the output of the LV bypass. Below a critical level, coronary perfusion pressure adversely affects LV contractility. ${ }^{18}$ In such a situation, the $E_{\text {es }}$ of the native heart estimated during LV bypass might not be the same as the $E_{\mathrm{es}}$ derived with the pump turned off, because deterioration in the coronary pressure and flow after weaning from $\mathrm{LV}$ bypass would depress the native heart function. The presence of this degree of ventricular dysfunction could be detected by decreases in $\mathrm{E}_{\mathrm{es}}$ as pumping rate is gradually lowered, because our method enables us to evaluate $\mathrm{E}_{\mathrm{es}}$ at each pumping rate. We did not specifically investigate this possibility in this study, and further experiments are needed to address diagnostic information potentially made available by pump rate changes.

Because we did not change native heart contractility, we cannot estimate the sensitivity of the measured $E_{\text {es }}$ in reflecting acute changes in LV contractility during LV bypass. Previous experimental and clinical experience has shown that $\mathrm{E}_{\mathrm{es}}$ can reasonably characterize acute changes in $\mathrm{LV}$ contractility, ${ }^{17}$ and we suspect that $\mathrm{E}_{\mathrm{es}}$ derived in this new manner would behave similarly. Additional study of $E_{e s}$ during LV bypass at increased and reduced contractilities, however, is needed to clarify this problem. In addition, we did not induce heart failure in this study. Validity of this method in the failing heart is needed in the experimental and clinical settings.

The analysis of ventricular function by the pressure-volume relationship provides a load-independent measure of LV contractility. However, volume measurement of the ventricle is important to achieve a reasonable ESPVR. We used ultrasonic crystals to calculate ventricular volume in this study. Implantation of the crystals is complicated, however, and not practical in a clinical setting. Although further development of transesophageal echocardiography would provide an LV volume measurement, the technique has not been established yet.
The recently developed conductance catheter enables on-line measurement of continuous pressurevolume data. ${ }^{19}$ At present the conductance catheter is the only option for instantaneous volume measurement in the clinical setting. We will have to determine the best techniques for obtaining pressure-volume data when we apply our method to an LV assist patient.

In conclusion, the $\mathrm{LV}$ contractility index, $\mathrm{E}_{\mathrm{es}}$, obtained without applying loading interventions during asynchronous LV bypass faithfully estimates native heart contractility.

The first author (O.K.) acknowledges through this study the continuous encouragement by Professor Hiroyuki Suga of the Second Department of Physiology of Okayama University Medical School and Professor Toshio Abe of the Department of Cardiothoracic Surgery of Nagoya University of Medicine. We also appreciate the excellent help by G. Allen Prophet, Cindy Miller, David N. Katz, and Mark Schwartz in experimental preparation.

\section{REFERENCES}

1. Pennington DG, Samuels LD, Williams G, et al. Experience with the Pierce-Donachy ventricular assist device in postcardiotomy patients with cardiogenic shock. World J Surg 1985;9:37-46.

2. Miller CA, Pae WE, Pierce WE. Combined registry for the clinical use of mechanical ventricular assist devices: postcardiotomy cardiogenic shock. ASAIO Trans 1990;36:43-6.

3. Pae WE, Miller CA, Matthews Y, Pierce WS. Ventricular assist devices for postcardiotomy cardiogenic shock: combined registry experience. J THORAC CARDIOVASC SURG 1992;104:541-53.

4. Verani MS, Sekela ME, Mahmarian, et al. Left ventricular function in patients with centrifugal left ventricular assist device. ASAIO Trans 1989;35:544-7.

5. Termuhlen DF, Swarts MT, Ruzevich SA, Reedy JE, Pennington DG. Hemodynamic predictors for weaning patients from ventricular assist devices (VADs). J Biomater Appl 1990;4:374-90.

6. Teruhlen DF, Swartz MT, Pennington DG, et al. Predictors for weaning patients from ventricular assist devices. ASAIO Trans 1988;34:131-9.

7. Magovern GJ, Golding LAR, Oyer PE, Cabrol C. Weaning and bridging. Ann Thorac Surg 1989;47: 102-7.

8. Suga $H$, Sagawa K. Instantaneous pressure-volume relationships and their ratio in the excised, supported canine left ventricle. Circ Res 1974;35:117-26.

9. Suga H, Sagawa K, Shoukas AA. Load independence of the instantaneous pressure-volume ratio of the canine left ventricle and effect of heart rate on the ratio. Circ Res 1973;32:314-22.

10. Kass DA, Yamazaki T, Burkoff D, Maughan WL, 
Sagawa K. Determination of left ventricular endsystolic pressure-volume relationships by the conductance (volume) catheter technique. Circulation 1986; 73:586-95.

11. Baan J, Van Der Velde E. Sensitivity of left ventricular end-systolic pressure-volume relation to type of loading intervention in dogs. Circ Res 1988;62:124758.

12. Guspta KB, Bavaria JE, Ratcliffe MB, Edmunds LH, Bogen DK. Measurement of end-systolic pressurevolume relations by intra-aortic balloon occlusion. Circulation 1989;80:1016-28.

13. Goto Y, Yamamoto J, Saito M, et al. Effect of right ventricular ischemia on left ventricular geometry and the end-diastolic pressure-volume relationship in the dog. Circulation 1985;72:1104-14.

14. Little WC, O'Rourke RA. Effect of regional ischemia on the left ventricular end-systolic pressure-volume relation in chronically instrumented dog. J Am Coll Cardiol 1985;5:297-302.
15. Nakamura T, Hayashi K, Seki J, et al. Effect of drive model of ventricular assist device on the left ventricular mechanics. Artif Organs 1988;12:56-66.

16. Baan J, Van Der Velde ET. Sensitivity of left ventricular end-systolic pressure-volume relation to type of loading intervention in dogs. 1988;62:1247-58.

17. Kass DA, Marino P, Maughan WL, Sagawa K. Determinants end-systolic pressure-volume relations during acute regional ischemia in situ. Circulation 1989;80: 1783-94.

18. Sunagawa K, Maughan WL, Frieshinger G, Guzman $\mathrm{P}$, Chang MS, Sagawa K. Effects of coronary arterial pressure on left ventricular end-systolic pressurevolume relation of isolated canine heart. Circ Res 1982;50:727-34.

19. Kass DA, Midei M, Graves W, Brinker JA, Maughan WL. Use of conductance catheter and transient inferior vena caval occlusion for rapid determination of pressure-volume relationships in man. Cathet Cardiovasc Diagn 1988;15:192-202. 